\title{
Biosimilars in Treatment of Multiple Sclerosis in Iran
}

\author{
Abdorreza Naser Moghadasi, $\mathrm{MD}^{1^{*}}$ \\ ${ }^{1}$ Multiple Sclerosis Research Center, Neuroscience Institute, Tehran University of Medical Sciences, Tehran, Iran
}

\begin{abstract}
Background: Biological drugs are manufactured via some changes made to the living organisms by genetic engineering. Notably, biological drugs are very expensive and their importation can impose economic pressure, especially on poorer countries. Thereafter, manufacturing these drugs has been considered by policymakers in many countries, resulting in the production of biosimilars. Iran requires a wide range of biological drugs due to the growing number of patients with multiple sclerosis. On the other hand, the poor economic situation of Iran due to repeated sanctions has had a great impact on the health care system, which has prevented the allocation of sufficient financial resources in this regard. Therefore, manufacturing biosimilar drugs due to their lower cost has received much attention in various fields of treatment.

Keywords: Biosimilars, Iran, Multiple sclerosis

Cite this article as: Naser Moghadasi A. Biosimilars in treatment of multiple sclerosis in iran. Arch Iran Med. 2021;24(10):779-782. doi: 10.34172/aim.2021.115
\end{abstract}

Received: August 24, 2020, Accepted: March 3, 2021, ePublished: October 1, 2021

\section{Introduction}

Multiple sclerosis (MS) is an autoimmune disease of the central nervous system that mainly affects young people. According to the published statistics and studies, the incidence of MS is increasing in Iran. ${ }^{1}$ Therefore, due to this increasing prevalence in Iran, more attention must be paid to the treatment of these patients. From 1993 up to the end of 2019, several drugs have entered the global drug market, most of which are classified as biological drugs. ${ }^{2}$ Biological drugs are manufactured via some changes made to the living organisms by genetic engineering. Notably, biological drugs are very expensive and can impose economic pressure, especially on poorer countries. Therefore, manufacturing these drugs has been considered by policymakers in many countries, resulting in the production of biosimilars. ${ }^{3}$ Biosimilars are biological drugs that are very similar in structure, function, side effects, and efficacy to the prototype. ${ }^{3}$

Iran needs to use a wide range of biological drugs due to the growing number of MS patients. In this regard, manufacturing biosimilar drugs due to their lower cost has received much attention in various fields of treatment. ${ }^{3}$

This article aimed to review biosimilar drugs produced and used in recent years in Iran to treat MS. Having a glance at these drugs and the studies conducted on them reveals the importance of biosimilar drugs in the treatment of patients with MS in Iran and reinforces the necessity of developing this industry.

\section{Biosimilars in Treatment of MS in Iran}

During past years, several biosimilar drugs have been produced by Iranian companies in Iran. By introducing these drugs, this section discusses studies performed on their different aspects. Unfortunately, despite the production of various biosimilar drugs in Iran, a limited number of studies have been conducted on them.

\section{CinnoVex}

CinnoVex is the first biosimilar produced in Iran for MS. It is an intramuscular and biosimilar interferon beta-1a drug developed by CinnaGen in collaboration with the Fraunhofer Institute in Germany in 2006. Currently, CinnoVex is used by nearly 11000 patients. ${ }^{4}$ Nafissi et al compared the efficacy and side effects of CinnoVex with Avonex, and after two years of monitoring patients, they reported no significant difference in terms of effectiveness and side effects between these two drugs. ${ }^{5}$

In a similar study by Pakdaman et al, 182 patients were randomly assigned to be treated with either CinnoVex or Avonex and then followed up for four and a half years. This study also showed no difference between these two drugs in terms of complication rate, magnetic resonance imaging (MRI), and clinical results. ${ }^{6}$

In another study, the cost-effectiveness of CinnoVex was compared with that of Avonex, and it was found that the cost per person for CinnoVex was $\$ 2410$ compared to $\$ 4515$ for Avonex, showing a significant difference. ${ }^{7}$ Later, these two drugs were compared regarding other aspects. For example, Hatem et al found no difference in terms of quality of life among the consumers of Avonex and CinnoVex. ${ }^{8}$ In addition, Abolfazli et al observed no difference in quality of life between CinnoVex and Avonex recipients after 30 months of follow-up. ${ }^{9}$

Shahkarami et al compared the levels of neutralizing 
antibodies (NAbs) in patients consuming these two drugs. Correspondingly, these patients were monitored for a 24-month period and had their NAbs measured every 6 months. Also, the amounts of antibodies were separately evaluated in two different laboratories, one in Iran and the other in Canada. The results for both drugs were similar in both laboratories, indicating that CinnoVex and Avonex have identical immunogenetic profiles in addition to having similar efficacy and safety. ${ }^{10}$

In another study on the level of cytokines in CinnoVex recipients and its relationship with patients' responses to treatment, it was found that the IFN- $\gamma$ level was significantly higher in the responder group compared to the non-responder group. ${ }^{11}$

\section{ReciGen}

ReciGen is a biosimilar product modeled after Rebif (interferon beta-1a subcutaneous) which was produced in 2009 by CinnaGen. Etemadifar et al compared ReciGen with Rebif and found no meaningful difference between these two drugs in terms of effectiveness and side effects. ${ }^{12}$

In a study conducted by Shokrollahi et al, the recipients of ReciGen and Rebif were studied for the amount of NAbs, and as a result, it was found that there was no significant difference between the consumers of these two drugs in terms of neutralizing antibody positivity. ${ }^{13}$

Unfortunately, despite the high consumption of this drug in Iran, few studies have evaluated the effect of ReciGen on MS patients. In 2020, following the use of interferons in the treatment of COVID-19, three studies examined the effectiveness of ReciGen in the treatment of COVID-19 indicating that the use of ReciGen could significantly reduce the severity of COVID-19..$^{14-16}$ An observational study examined the effect of ReciGen on the incidence, hospitalization, and mortality in 75 MS patients taking the drug. Correspondingly, the mean duration of ReciGen consumption was 6 years in these patients. Among them, one patient was infected with COVID-19 who did not need hospitalization and whose symptoms rapidly improved within five days. This study showed that although the use of ReciGen in these patients did not reduce the incidence of COVID-19, the rates of hospitalization and death were significantly decreased. ${ }^{17}$

\section{Ziferon}

Ziferon is another biosimilar produced by Zist Daru Danesh Company in 2010, which is modeled after Betaferon (interferon beta-1 $\beta$ ). In order to examine the structural property of this biologic drug, Dadgarnejad et al used the micellar electrokinetic chromatography (MEKC) method and found that Ziferon has an acceptable potency comparable with its brand. ${ }^{18}$

In a study by Gheini et al, the effectiveness of these two drugs was compared in terms of disease progression, the number of attacks, the effect on MRI, and their side effects over a two-year period, and no significant difference was observed between them. ${ }^{19}$ In another study conducted in Isfahan, the authors showed that $9 \%$ of patients who enrolled in the study had received Ziferon as the main drug which was comparable with other high dose interferons including ReciGen and Betaferon. ${ }^{20}$ The effectiveness of Ziferon as a combination therapy on patients with COVID-19 has been studied in $\operatorname{Iran}^{21}$ and the results of this study will be published in the future which may increase our knowledge regarding the efficacy and safety of Ziferon.

\section{Zytux}

Besides interferons, monoclonal antibody drugs have also attracted the attention of Iranian pharmacists and have been developed to be used for various diseases. The only biosimilar monoclonal antibody drug, which is available in Iran and is widely used for MS patients, is called Zytux. This drug is actually the MabThera biosimilar (rituximab) which was produced in 2010 by AryoGen Company. ${ }^{22}$

The effectiveness and safety of this drug were compared with MabThera in a study on patients with chronic lymphocytic leukemia (CLL). In the mentioned doubleblind study, 70 patients were divided into two equal groups as follows: one group received Zytux and the other one received MabThera. No significant difference was observed between the two groups in terms of drug efficacy and side effects. ${ }^{23}$

In another observational study, 10 patients with CLL and 10 patients with non-Hodgkin lymphoma were treated by Zytux and were followed up for 6 months. In this study, the efficacy and safety of Zytux were acceptable and were comparable with reports of MabThera in the literature. ${ }^{24}$ Toosi et al investigated the safety and efficacy of this biosimilar drug in patients with pemphigus vulgaris and reported desirable outcomes in this regard..$^{25}$

Torkashvand et al compared the binding affinity of Fc gamma receptors between Zytux and MabThera. Fc gamma receptors are the most important receptors mediating the antibody-dependent cell-mediated cytotoxicity and complement-dependent cytotoxicity. These two pathways are the main ways through which rituximab affects diseases. The authors showed that there was not any significant difference between these two drugs in terms of the binding affinity of Fc gamma receptors. ${ }^{26}$

Although Zytux is widely used in treatment of MS patients in Iran, only one observational study has been performed on its efficacy and side effects. ${ }^{22}$ This study was conducted on 100 MS patients taking Zytux (20 patients had relapsing-remitting MS, 20 patients had primary progressive MS, and 60 patients had secondary progressive MS). It was found that Zytux has no serious side effects and is effective on all types of MS.

Similarly, Naser Moghadasi and Ghoreyshi Tayyebi have reported a 21-year-old female with highly active MS who was effectively treated by Zytux. ${ }^{27}$

Another observational study examined the outcome of pregnancy in MS patients who became pregnant while taking Zytux. Of 21 pregnancies, 8 pregnancies resulted 
in term births and two pregnancies resulted in preterm births. Two spontaneous abortions and 7 miscarriages were performed either in response to the suggestion of their physician or by the request of the mother. Overall, the study showed that despite taking Zytux, pregnancy can be safe for both mother and infant. ${ }^{28}$

In another study, short-term and long-term side effects of Zytux were studied on patients with MS and neuromyelitis optica spectrum disorder, revealing that these side effects were usually mild, except for one case of bradycardia. ${ }^{29}$

\section{Discussion}

Biosimilars are widely used in Iran. Extensive use of biosimilars has practically resulted in using various drugs with reasonable prices by MS patients. Although the number of conducted studies in this field is limited, it seems that Iranian companies have been successful in manufacturing these biosimilars, and the biosimilar sample is identical to the original sample in terms of effectiveness and safety. However, a better understanding of the efficacy of these drugs requires conducting further comprehensive studies. With the exception of CinnoVex that has been assessed from different perspectives to some extent, such studies have not been performed on the other biosimilars despite their widespread use, which makes it difficult to evaluate these drugs. Lack of numerous studies is the most concerning point regarding the wide spread use of biosimilars in Iran that must be immediately addressed. Another crucial issue is the growth of pharmaceutical companies manufacturing monoclonal antibody drugs. As mentioned earlier, the only biosimilar monoclonal antibody drug used to treat MS in Iran is Zytux. Iranians' access to other monoclonal drugs that are not biosimilars, is practically limited due to their high prices. Also, several drugs such as Ocrelizumab, Alemtuzumab, and Natalizumab can be used widely in treatment of MS patients, if they are reasonably priced. Thus, Iranian pharmaceutical companies should plan to manufacture biosimilars of the mentioned drugs.

\section{Conflict of Interest Disclosures}

Abdorreza Naser Moghadasi has received speaker's honoraria from CinnaGen and AryoGen Companies.

He has collaborated with CinnaGen and Zist Daru Danesh Companies concerning conducting clinical trials.

\section{Ethical Statement}

Not applicable.

\section{References}

1. Sahraian MA, Sahebkar M, Dehghani R, Derakhshan-Jazari M, Kazami-Moghaddam V, Kouchaki E. Multiple sclerosis-a disease on a dramatically rising trend in Iran: review of possible reasons. Iran J Neurol. 2017;16(1):34-40.

2. Rivera VM. Multiple sclerosis: a global concern with multiple challenges in an era of advanced therapeutic complex molecules and biological medicines. Biomedicines. 2018;6(4):112. doi: 10.3390/biomedicines6040112.

3. Cheraghali AM. Biosimilars; a unique opportunity for Iran national health sector and national pharmaceutical industry. Daru. 2012;20(1):35. doi: 10.1186/2008-2231-20-35.

4. Naser Moghadasi A. History of multiple sclerosis in Iran. Arch Iran Med. 2020;23(3):211-5.

5. Nafissi S, Azimi A, Amini Harandi A, Salami S, Shahkarami MA, Heshmat R. Comparing efficacy and side effects of a weekly intramuscular biogeneric/biosimilar interferon beta-1a with Avonex in relapsing remitting multiple sclerosis: a double blind randomized clinical trial. Clin Neurol Neurosurg. 2012;114(7):986-9. doi: 10.1016/j.clineuro.2012.02.039.

6. Pakdaman H, Abbasi M, Gharagozli K, Ashrafi F, Delavar Kasmaei $\mathrm{H}$, Amini Harandi A. A randomized double-blind trial of comparative efficacy and safety of Avonex and CinnoVex for treatment of relapsing-remitting multiple sclerosis. Neurol Sci. 2018;39(12):2107-13. doi: 10.1007/s10072-018-3550-8.

7. Najafi B, Ghaderi H, Jafari M, Najafi S, Ahmad Kiadaliri A. Cost effectiveness analysis of Avonex and CinnoVex in relapsing remitting MS. Glob J Health Sci. 2014;7(2):139-47. doi: 10.5539/gjhs.v7n2p139.

8. Hatam N, Bastani P, Shahtaheri RS. Quality of life in relapsingremitting multiple sclerosis patients receiving CinnoVex compared with Avonex. J Res Pharm Pract. 2016;5(3):181-5. doi: 10.4103/2279-042x.185725.

9. Abolfazli R, Hosseini A, Gholami K, Javadi MR, Torkamandi $\mathrm{H}$, Emami S. Quality of life assessment in patients with multiple sclerosis receiving interferon beta-1a: a comparative longitudinal study of Avonex and its biosimilar CinnoVex. ISRN Neurol. 2012;2012:786526. doi: 10.5402/2012/786526.

10. Shahkarami MA, Vaziri B, Salami S, Amini Harandi A, Oger J. Neutralizing antibodies in multiple sclerosis patients on weekly intramuscular Avonex and biosimilar interferon beta1a (CinnoVex): comparing results of measurements in two different laboratories. J Immunol Methods. 2013;388(1-2):468. doi: 10.1016/j.jim.2012.11.013.

11. Taheri M, Ghafouri-Fard S, Solgi G, Sayad A, Mazdeh M, Omrani MD. Determination of cytokine levels in multiple sclerosis patients and their relevance with patients' response to Cinnovex. Cytokine. 2017;96:138-43. doi: 10.1016/j. cyto.2017.04.007.

12. Etemadifar M, Soheilnader S, Shahkarami MA, Mehrabi Kooshki A. Comparison of the efficacy and side effects of IFN beta-1a (Rebif) and a biosimilar product (ReciGen) in patients with multiple sclerosis. J Isfahan Med Sch. 2012;29(162):196474. [Persian].

13. Shokrollahi Barough $M$, Ashtari F, Sadat Akhavi M, Asghari N, Mosayebi G, Mirmohammadkhani M, et al. Neutralizing antibody production against Rebif $\left({ }\right.$ and ReciGen $\left({ }^{\circ}\right.$ in relapsing-remitting multiple sclerosis (RRMS) patients and its association with patient's disability. Int Immunopharmacol. 2018;62:109-13. doi: 10.1016/j.intimp.2018.06.032.

14. Davoudi-Monfared E, Rahmani $\mathrm{H}$, Khalili $\mathrm{H}$, Hajiabdolbaghi $M$, Salehi M, Abbasian L, et al. A randomized clinical trial of the efficacy and safety of interferon beta-1a in treatment of severe COVID-19. Antimicrob Agents Chemother. 2020;64(9). doi: 10.1128/aac.01061-20.

15. Payandemehr P, Azhdarzadeh M, Bahrami-Motlagh $\mathrm{H}$, Hadadi A, Najmeddin F, Shahmirzaei S, et al. Interferon beta-1a as a candidate for COVID-19 treatment; an open-label single-arm clinical trial. Front Emerg Med. 2020;4(2 Suppl):e51.

16. Dastan F, Nadji SA, Saffaei A, Marjani M, Moniri A, Jamaati $\mathrm{H}$, et al. Subcutaneous administration of interferon beta1a for COVID-19: a non-controlled prospective trial. Int Immunopharmacol. 2020;85:106688. doi: 10.1016/j. intimp.2020.106688.

17. Naser Moghadasi A, Anjidani N. Evaluation of the incidence, severity, and mortality rate of COVID-19 in patients with multiple sclerosis receiving interferon beta-1a. Front Emerg Med. 2021;5(2):e16. doi: 10.18502/fem.v5i2.5607. 
18. Dadgarnejad $M$, Rastegar H, Ilka H, Shekarchi M, Adib N, Alebouyeh $\mathrm{M}$, et al. Development and validation of a micellar capillary electrophoresis method for determination of IFN $\beta$ $1 \mathrm{~b}$ in lyophilized formulation of a biosimilar product. Iran J Pharm Res. 2015;14(3):747-55.

19. Gheini MR, Sahraian MA, Azimi AR, Naser Moghadasi A, Abdoli M, Rahimi G, et al. Comparing the safety and efficacy of Ziferon and Betaferon in patients with remitting-relapsing multiple sclerosis. Pharm Biomed Res. 2019;5(4):21-6. doi: 10.18502/pbr.v5i4.2393.

20. Rahimi F, Rasekh H, Peiravian F, Abbasian E. Using discrete choice experiment to determine willingness to pay for medicine interferon-beta by multiple sclerosis patients. Iran J Pharm Sci. 2018;14(4):71-8. doi: 10.22034/ijps.2018.37550.

21. Naghibi Irvani SS, Golmohammadi M, Pourhoseingholi MA, Shokouhi S, Alavi Darazam I. Effectiveness of interferon beta$1 \mathrm{a}$, compared to interferon beta- $1 \mathrm{~b}$ and the usual therapeutic regimen to treat adults with moderate to severe COVID-19: structured summary of a study protocol for a randomized controlled trial. Trials. 2020;21(1):473. doi: 10.1186/s13063020-04382-3.

22. Naser Moghadasi A, Darki A, Masoumi P, Hashemi SN, Ghadiri F. Evaluating the efficacy and safety of Zytux ${ }^{\top M}$ (rituximab, AryoGen pharmed) in Iranian multiple sclerosis patients: an observational study. Mult Scler Relat Disord. 2019;36:101419. doi: 10.1016/j.msard.2019.101419.

23. Toogeh G, Faranoush M, Razavi SM, Jalaeikhoo H, Allahyari A, Ravanbod MR, et al. A double-blind, randomized comparison study between Zytux ${ }^{\mathrm{TM}}$ vs MabThera ${ }^{\circledR}$ in treatment of CLL with FCR regimen: non-inferiority clinical trial. Int J Hematol Oncol Stem Cell Res. 2018;12(2):84-91.
24. Alwan AF, Abdulsahib MA, Abbas DD, Abdulsattar SA Ensaif RT. Efficacy and safety of biosimilar rituximab (Zytux ${ }^{\mathrm{TM}}$ ) in newly diagnosed patients with non-Hodgkin lymphoma and chronic lymphocytic leukemia. Hematol Rep. 2020;12(3):8296. doi: 10.4081/hr.2020.8296.

25. Toosi R, Mahmoudi H, Balighi K, Teimourpour A, Alaeen $H$, Shaghaghi $M$, et al. Efficacy and safety of biosimilar rituximab in patients with pemphigus vulgaris: a prospective observational study. J Dermatolog Treat. 2021;32(1):33-40. doi: 10.1080/09546634.2019.1617831.

26. Torkashvand F, Vaziri B, Saadatirad A, Reddy GS, Srinivasulu K, Mahendran B, et al. FC gamma receptors binding affinity analysis of an approved rituximab in Iran and the originator rituximab. Acad J Biotechnol. 2018;7(1):14-20. doi: 10.15413/ ajb.2018.0126.

27. Naser Moghadasi A, Ghoreyshi Tayyebi E. Management of high disease activity in multiple sclerosis using Zytux ${ }^{\mathrm{TM}}$ (rituximab, AryoGen pharmed): evidence from a case report. J Kermanshah Univ Med Sci. 2020;24(1):e101422. doi: 10.5812/jkums.101422.

28. Seyed Ahadi M, Sahraian MA, Baghbanian SM, Azimi A, Shaygannejad V, Anjidani N, et al. Pregnancy outcome in patients with multiple sclerosis treated with rituximab: a caseseries study. Mult Scler Relat Disord. 2021;47:102667. doi: 10.1016/j.msard.2020.102667.

29. Farzadfard F, Poursadeghfard $M$, Ashjazadeh N. Infusionrelated, short-term, and delayed side effects of rituximab in multiple sclerosis and neuromyelitis optica spectrum disorder. Shiraz E-Med J. 2020;22(3):e101625. doi: 10.5812/ semj.101625. 